\title{
Starlikeness of Analytic Functions with Subordinate Ratios
}

\author{
Rosihan M. Ali, ${ }^{1}$ Vaithiyanathan Ravichandran $\mathbb{D}^{2},{ }^{2}$ and Kanika Sharma $\mathbb{D}^{3}$ \\ ${ }^{1}$ School of Mathematical Sciences, Universiti Sains Malaysia, George Town 11800 USM, Penang, Malaysia \\ ${ }^{2}$ Department of Mathematics, National Institute of Technology, Tiruchirappalli 620015, India \\ ${ }^{3}$ Department of Mathematics, Atma Ram Sanatan Dharma College, University of Delhi, Delhi 110021, India
}

Correspondence should be addressed to Vaithiyanathan Ravichandran; vravi68@gmail.com

Received 31 July 2021; Accepted 9 October 2021; Published 29 October 2021

Academic Editor: Ming-Sheng Liu

Copyright ( $\odot 2021$ Rosihan M. Ali et al. This is an open access article distributed under the Creative Commons Attribution License, which permits unrestricted use, distribution, and reproduction in any medium, provided the original work is properly cited.

Let $h$ be a nonvanishing analytic function in the open unit disc with $h(0)=1$. Consider the class consisting of normalized analytic functions $f$ whose ratios $f(z) / g(z), g(z) / z p(z)$, and $p(z)$ are each subordinate to $h$ for some analytic functions $g$ and $p$. The radius of starlikeness of order $\alpha$ is obtained for this class when $h$ is chosen to be either $h(z)=\sqrt{1+z}$ or $h(z)=e^{z}$. Further, starlikeness radii are also obtained for each of these two classes, which include the radius of Janowski starlikeness, and the radius of parabolic starlikeness.

\section{Two Subclasses of Normalized Analytic Functions}

Let $\mathscr{A}$ denote the class of normalized analytic functions $f(z)=z+\sum_{k=2}^{\infty} a_{k} z^{k}$ in the unit disc $\mathbb{D}=\{z \in \mathbb{C}:|z|<1\}$. A prominent subclass of $\mathscr{A}$ is the class $\mathcal{S}^{*}$ consisting of functions $f \in \mathscr{A}$ such that $f(\mathbb{D})$ is a starlike domain with respect to the origin. Geometrically, this means the linear segment joining the origin to every other point $w \in f(\mathbb{D})$ lies entirely in $f(\mathbb{D})$. Every starlike function in $\mathscr{A}$ is necessarily univalent.

Since $f^{\prime}(0)$ does not vanish, every function $f \in \mathscr{A}$ is locally univalent at $z=0$. Further, each function $f \in \mathscr{A}$ mirrors the identity mapping near the origin and thus, in particular, maps small circles $|z|=r$ onto curves which bound starlike domains. If $f \in \mathscr{A}$ is also required to be univalent in $\mathbb{D}$, then it is known that $f$ maps the disc $|z|<r$ onto a domain starlike with respect to the origin for every $r \leq r_{0}:=\tan h(\pi / 4)$ (see [1], Corollary, p. 98). The constant $r_{0}$ cannot be improved. Denoting by $\delta$ the class of univalent functions $f \in \mathscr{A}$, the number $r_{0}=\tan h(\pi / 4)$ is commonly referred to as the radius of starlikeness for the class $\mathcal{S}$.

Another informative description of the class $\mathcal{S}$ is its radius of convexity. Here, it is known that every $f \in \mathcal{S}$ maps the disc $|z|<r$ onto a convex domain for every $r \leq r_{0}:=2-\sqrt{3}([1]$, Corollary, p. 44). Thus, the radius of convexity for $\mathcal{S}$ is $r_{0}=2-\sqrt{3}$.

To formulate a radius description for other entities besides starlikeness and convexity, consider in general two families $\mathscr{G}$ and $\mathscr{M}$ of $\mathscr{A}$. The $\mathscr{G}$-radius for the class $\mathscr{M}$, denoted by $R_{\mathscr{G}}(\mathscr{M})$, is the largest number $R$ such that $r^{-1} f(r z) \in \mathscr{G}$ for every $0<r \leq R$ and $f \in \mathscr{M}$. Thus, for example, an equivalent description of the radius of starlikeness for $\mathcal{S}$ is that the $\mathcal{S}^{*}$-radius for the class $\mathcal{S}$ is $R_{\mathcal{S}^{*}}(\mathcal{S})=\tanh (\pi / 4)$.

In this paper, we seek to determine the radius of starlikeness and certain other $\mathscr{G}$-radius, for particular subclasses $\mathscr{G}$ of $\mathscr{A}$. Several widely studied subclasses of $\mathscr{A}$ have simple geometric descriptions; these functions are often expressed as a ratio between two functions. Among the very early studies in this direction is the class of close-to-convex functions introduced by Kaplan [2] and Reade's class [3] of close-to-starlike functions. Close-to-convex functions are necessarily univalent, but not so for close-to-starlike functions.

In this paper, we examine two different subclasses of functions in $\mathscr{A}$ satisfying a certain subordination of ratios. Interestingly, these classes contain nonunivalent functions. An analytic function $f$ is subordinate to an analytic function $g$, written $f<g$, if 


$$
f(z)=g(w(z)), \quad z \in \mathbb{D},
$$

for some analytic self-map $w$ in $\mathbb{D}$ with $|w(z)| \leq|z|$. The function $w$ is often referred to as a Schwarz function.

Now, let $h$ be a nonvanishing analytic function in $\mathbb{D}$ with $h(0)=1$. The classes treated in this paper consist of functions $f \in \mathscr{A}$ whose ratios $f(z) / g(z), g(z) / z p(z)$, and $p(z)$ are each subordinate to $h$ for some analytic functions $g$ and $p$ :

$$
\begin{aligned}
\frac{f(z)}{g(z)} & \prec h(z), \\
\frac{g(z)}{z p(z)} & \prec h(z), \\
p(z) & \prec h(z) .
\end{aligned}
$$
by

$$
\mathscr{T}_{1}:=\left\{f \in \mathscr{A}: \frac{f(z)}{g(z)}<\sqrt{1+z}, \frac{g(z)}{z p(z)}<\sqrt{1+z} \text {, for some } g \in \mathscr{A}, p(z) \prec \sqrt{1+z}\right\} \text {. }
$$

$$
\begin{aligned}
& f_{1}(z)=z(1+z)^{3 / 2}, \\
& g_{1}(z)=z(1+z), \\
& p_{1}(z)=\sqrt{1+z}
\end{aligned}
$$

Then, $f_{1}(z) / g_{1}(z) \prec \sqrt{1+z}$ and $g_{1}(z) / z p_{1}(z) \prec \sqrt{1+z}$, so that $f_{1} \in \mathscr{T}_{1}$. The function $f_{1}$ will be shown to play the role of an extremal function for the class $\mathscr{T}_{1}$. Since $f_{1}^{\prime}$ vanishes at $z=-2 / 5$, the function $f_{1}$ is nonunivalent, and thus, the class $\mathscr{T}_{1}$ contains nonunivalent functions. Incidentally, $f_{1}$ demonstrates the radius of univalence for $\mathscr{T}_{1}$ is at most $2 / 5$. In Theorem 1 , the radius of starlikeness for $\mathscr{T}_{1}$ is shown to be $2 / 5$, whence $\mathscr{T}_{1}$ has radius of univalence $2 / 5$.

The following is a useful result in investigating the starlikeness of the class $\mathscr{T}_{1}$.

Lemma 1. Let $p(z) \prec \sqrt{1+z}$. Then, $p$ satisfies the sharp inequalities

$$
\begin{aligned}
\sqrt{1-r} & \leq|p(z)| \leq \sqrt{1+r}, \quad|z| \leq r, \\
\left|\frac{z p^{\prime}(z)}{p(z)}\right| & \leq \frac{r}{2(1-r)}, \quad|z| \leq r .
\end{aligned}
$$

Proof. If $p(z)<\sqrt{1+z}$, then $p^{2}(z)=1+w(z)$ for some Schwarz function $w$. The well-known Schwarz lemma shows that $|w(z)| \leq|z|$ and

$$
\left|w^{\prime}(z)\right| \leq \frac{1-|w(z)|^{2}}{1-|z|^{2}}
$$

Therefore,

When $p$ is the constant one function, then the class contains functions $f \in \mathscr{A}$ satisfying the subordination of ratios

$$
\begin{aligned}
& \frac{f(z)}{g(z)} \prec h(z), \\
& \frac{g(z)}{z} \prec h(z) .
\end{aligned}
$$

$$
|p(z)|^{2}=|1+w(z)| \leq 1+|w(z)| \leq 1+|z| \leq 1+r,
$$

for $|z| \leq r$, that is, $|p(z)| \leq \sqrt{1+r}$ for $|z| \leq r$. Similarly, $|p(z)| \geq \sqrt{1-r}$ for $|z| \leq r$.

Since $2 z p^{\prime}(z) / p(z)=z w^{\prime}(z) /(1+w(z))$, the inequality (8) readily shows

$$
\begin{aligned}
2\left|\frac{z p^{\prime}(z)}{p(z)}\right| & \leq \frac{|z|\left|w^{\prime}(z)\right|}{1-|w(z)|} \leq \frac{|z|(1+|w(z)|)}{1-|z|^{2}} \\
& \leq \frac{|z|(1+|z|)}{1-|z|^{2}}=\frac{|z|}{1-|z|} \leq \frac{r}{1-r}
\end{aligned}
$$

for $|z| \leq r$. This proves (7). The inequalities are sharp for the function $p: \mathbb{D} \longrightarrow \mathbb{C}$ defined by $p(z)=\sqrt{1+z}$.

For $f \in \mathscr{T}_{1}$, let $p_{1}(z)=f(z) / g(z)$ and $p_{2}(z)=$ $g(z) / z p(z)$. Then, $f(z)=z p(z) p_{1}(z) p_{2}(z)$ and

$$
\left|\frac{z f^{\prime}(z)}{f(z)}-1\right| \leq\left|\frac{z p^{\prime}(z)}{p(z)}\right|+\left|\frac{z p_{1}^{\prime}(z)}{p_{1}(z)}\right|+\left|\frac{z p_{2}^{\prime}(z)}{p_{2}(z)}\right| .
$$

Since $p, p_{1}, p_{2} \prec \sqrt{1+z}$, we deduce from (7) and (11) that

$$
\left|\frac{z f^{\prime}(z)}{f(z)}-1\right| \leq \frac{3 r}{2(1-r)}, \quad|z| \leq r,
$$

for each function $f \in \mathscr{T}_{1}$. Sharp growth inequalities also follow from (6):

$$
r(1-r)^{3 / 2} \leq|f(z)| \leq r(1+r)^{3 / 2}
$$


for each $f \in \mathscr{T}_{1}$. Crude distortion inequalities can readily be obtained from (12) and the growth inequality; however, finding sharp estimates remain an open problem.

The class $\mathscr{T}_{2}$ : this class is defined by

$$
\mathscr{T}_{2}:=\left\{f \in \mathscr{A}: \frac{f(z)}{g(z)}<e^{z}, \frac{g(z)}{z p(z)}<e^{z} \text {, for some } g \in \mathscr{A}, p(z)<e^{z}\right\} .
$$

Let $f_{2}, g_{2}, p_{2}: \mathbb{D} \longrightarrow \mathbb{C}$ be given by

$$
\begin{aligned}
& f_{2}(z)=z e^{3 z}, \\
& g_{2}(z)=z e^{2 z}, \\
& p_{2}(z)=e^{z}
\end{aligned}
$$

Evidently, $f_{2}(z) / g_{2}(z)<e^{z}, g_{2}(z) / z p_{2}(z)<e^{z}$, so that $f_{2} \in \mathscr{T}_{2}$, and the class $\mathscr{T}_{2}$ is nonempty. Similar to $f_{1} \in \mathscr{T}_{1}$, the function $f_{2}$ plays the role of an extremal function for the class $\mathscr{T}_{2}$. The Taylor series expansion for $f_{2}$ is

$$
f_{2}(z)=z+3 z^{2}+\frac{9 z^{3}}{2}+\frac{9 z^{4}}{2}+\frac{27 z^{5}}{8}+\cdots
$$

Comparing the second coefficient, it is clear that $f_{2}$ is nonunivalent. Hence, the class $\mathscr{T}_{2}$ contains nonunivalent functions. The derivative $f_{2}^{\prime}$ vanishes at $z=-1 / / 3$, which shows the radius of univalence for $\mathscr{T}_{2}$ is at most $1 / 3$. From Theorem 1, the radius of starlikeness is shown to be $1 / 3$, and so the radius of univalence for $\mathscr{T}_{2}$ is $1 / 3$.

Lemma 2. Every $p(z)<e^{z}$ satisfies the sharp inequalities

$$
\begin{aligned}
& e^{-r} \leq|p(z)| \leq e^{r}, \quad|z| \leq r, \\
& \left|\frac{z p^{\prime}(z)}{p(z)}\right| \leq \begin{cases}r, & |z| \leq r \leq \sqrt{2}-1, \\
\frac{\left(1+r^{2}\right)^{2}}{4\left(1-r^{2}\right)}, & |z|=r \geq \sqrt{2}-1 .\end{cases}
\end{aligned}
$$

Proof. Let $p(z)<e^{z}$. Since $p(z)=e^{w(z)}$ for some Schwarz self-map $w$ satisfying $|w(z)| \leq|z|$, it follows that

$$
e^{-|z|} \leq e^{-|w(z)|} \leq|p(z)|=e^{\operatorname{Re} w(z)} \leq e^{|w(z)|} \leq e^{|z|} .
$$

The inequalities become equality for the function $p: \mathbb{D} \longrightarrow \mathbb{C}$ defined by $p(z)=e^{z}$ respectively at $z=-r$ and $z=r$.

The function $w$ also satisfies the sharp inequality (see [1], Corollary, p. 199)

$$
\left|w^{\prime}(z)\right| \leq \begin{cases}1, & r=|z| \leq \sqrt{2}-1, \\ \frac{\left(1+r^{2}\right)^{2}}{4 r\left(1-r^{2}\right)}, & r \geq \sqrt{2}-1 .\end{cases}
$$

From $z p^{\prime}(z) / p(z)=z w^{\prime}(z)$, we conclude that

$$
\left|\frac{z p^{\prime}(z)}{p(z)}\right| \leq \begin{cases}r, & r=|z| \leq \sqrt{2}-1, \\ \frac{\left(1+r^{2}\right)^{2}}{4\left(1-r^{2}\right)}, & r \geq \sqrt{2}-1 .\end{cases}
$$

This inequality is sharp for the function $p: \mathbb{D} \longrightarrow \mathbb{C}$ defined by $p(z)=e^{z}$ when $r=|z| \leq \sqrt{2}-1$. It is also sharp in the remaining interval for the function $p(z)=e^{w(z)}$, where $w$ is the extremal function for which equality holds in (20).

For $\quad f \in \mathscr{T}_{2}$, let $\quad p_{1}(z)=f(z) / g(z) \quad$ and $p_{2}(z)=g(z) / z p(z)$. Then, $f(z)=z p(z) p_{1}(z) p_{2}(z)$ and

$$
\left|\frac{z f^{\prime}(z)}{f(z)}-1\right| \leq\left|\frac{z p^{\prime}(z)}{p(z)}\right|+\left|\frac{z p_{1}^{\prime}(z)}{p_{1}(z)}\right|+\left|\frac{z p_{2}^{\prime}(z)}{p_{2}(z)}\right| .
$$

Since $p, p_{1}, p_{2}<e^{z}$, estimates (18) and (22) show that

$$
\left|\frac{z f^{\prime}(z)}{f(z)}-1\right| \leq \begin{cases}3 r, & r=(z) \leq \sqrt{2}-1, \\ \frac{3\left(1+r^{2}\right)^{2}}{4\left(1-r^{2}\right)}, & r \geq \sqrt{2}-1,\end{cases}
$$

for each function $f \in \mathscr{T}_{2}$. It also follows from (17) that

$$
r e^{-3 r} \leq|f(z)| \leq r e^{3 r}
$$

holds for each function $f \in \mathscr{T}_{2}$ and that these estimates are sharp.

In this paper, we shall adopt the commonly used notations for subclasses of $\mathscr{A}$. First, for $0 \leq \alpha<1$, let $\mathcal{S}^{*}(\alpha)$ denote the class of starlike functions of order $\alpha$ consisting of functions $f \in \mathscr{A}$ satisfying the subordination

$$
\frac{z f^{\prime}(z)}{f(z)}<\frac{1+(1-2 \alpha) z}{1-z} .
$$

Thus,

$$
\operatorname{Re} \frac{z f^{\prime}(z)}{f(z)}>\alpha, \quad z \in \mathbb{D} .
$$

The case $\alpha=0$ corresponds to the classical functions whose image domains are starlike with respect to the origin. Various other starlike subclasses of $\mathscr{A}$ occurring in the literature can be expressed in terms of the subordination

$$
\frac{z f^{\prime}(z)}{f(z)}<\varphi(z),
$$

for suitable choices of the superordinate function $\varphi$. When $\varphi: \mathbb{D} \longrightarrow \mathbb{C}$ is chosen to be $\varphi(z):=(1+A z) /(1+B z)$, $-1 \leq B<A \leq 1$, the subclass derived is denoted by $\mathcal{S}^{*}[A, B]$. Functions $f \in \mathcal{S}^{*}[A, B]$ are known as Janowski starlike functions. When $\varphi(z):=1+\left(2 / \pi^{2}\right)((\log ((1+\sqrt{z})$ $\left./(1-\sqrt{z})))^{2}\right)$, the subclass is denoted by $\mathcal{S}_{p}^{*}$, and its functions are called parabolic starlike functions. 
In Section 2 of this paper, the radius of starlikeness of order $\alpha$, Janowski starlikeness, and parabolic starlikeness are found for the classes $\mathscr{T}_{i}$, with $i=1,2$. Section 3 deals with the determination of the $\mathscr{G}$-radius for the class $\mathscr{T}_{i}$ with $i=1,2$, for certain other subclasses $\mathscr{G}$ occurring in the literature. These classes are associated with particular choices of the superordinate function $\varphi$ in (27). As mentioned earlier, the $\mathscr{G}$-radius for a given class $\mathscr{M}$, denoted by $R_{\mathscr{G}}(\mathscr{M})$, is the largest number $R$ such that $r^{-1} f(r z) \in \mathscr{G}$ for every $0<r \leq R$ and $f \in \mathscr{M}$. It will become apparent in the forthcoming proofs that there are common features in the methodology of finding the $\mathscr{G}$-radius for each of these subclasses.

\section{Starlikeness of Order $\alpha$, Janowski Starlikeness, and Parabolic Starlikeness}

The first result deals with the $\mathcal{S}^{*}(\alpha)$-radius (radius of starlikeness of order $\alpha$ ) for the classes $\mathscr{T}_{1}$ and $\mathscr{T}_{2}$. This radius is shown to equal the $\mathcal{S}_{\alpha}^{*}$-radius, where $\mathcal{S}_{\alpha}^{*}$ is the subclass containing functions $f \in \mathscr{A}$ satisfying $\left|z f^{\prime}(z) / f(z)-1\right|<1-\alpha$. The latter condition also implies that $\mathcal{S}_{\alpha}^{*} \subset \mathcal{S}^{*}(\alpha)$.

Theorem 1. Let $0 \leq \alpha<1$. The radii of starlikeness of order $\alpha$ for $\mathscr{T}_{1}$ and $\mathscr{T}_{2}$ are

(i) $R_{\mathcal{S}^{*}(\alpha)}\left(\mathscr{T}_{1}\right)=R_{\mathcal{S}_{\alpha}^{*}}\left(\mathscr{T}_{1}\right)=2(1-\alpha) /(5-2 \alpha)$,

(ii) $R_{\mathcal{S}^{*}(\alpha)}\left(\mathscr{T}_{2}\right)=R_{\mathcal{S}_{\alpha}^{*}}\left(\mathscr{T}_{2}\right)=(1-\alpha) / 3$.

Proof

(i) The function $\sigma(r)=(2-5 r) /(2-2 r)$ is a decreasing function on $[0,1)$. Further, the number $R_{1}:=2(1-$ $\alpha) /(5-2 \alpha)$ is the root of the equation $\sigma(r)=\alpha$. For $f \in \mathscr{T}_{1}$ and $0<r=|z| \leq R_{1}$, the inequality (12) readily yields

$$
\begin{gathered}
\operatorname{Re} \frac{z f^{\prime}(z)}{f(z)} \geq 1-\frac{3 r}{2(1-r)}=\frac{2-5 r}{2-2 r}=\sigma(r) \geq \sigma\left(R_{1}\right)=\alpha, \\
\left|\frac{z f^{\prime}(z)}{f(z)}-1\right| \leq \frac{3 r}{2(1-r)}=1-\sigma(r) \leq 1-\sigma\left(R_{1}\right)=1-\alpha .
\end{gathered}
$$

At $z=-R_{1}$, the function $f_{1} \in \mathscr{T}_{1}$ given by $f_{1}(z)=$ $z(1+z)^{3 / 2}$ yields

$$
\frac{z f_{1}^{\prime}(z)}{f_{1}(z)}=\frac{2+5 z}{2+2 z}=\frac{2-5 R_{1}}{2-2 R_{1}}=\alpha .
$$

Thus,

$$
\begin{gathered}
\operatorname{Re} \frac{z f_{1}^{\prime}(z)}{f_{1}(z)}=\alpha, \\
\left|\frac{z f_{1}^{\prime}(z)}{f_{1}(z)}-1\right|=1-\alpha .
\end{gathered}
$$

This proves that the $\mathcal{S}^{*}(\alpha)$ and $\mathcal{S}_{\alpha}^{*}$ radii for $\mathscr{T}_{1}$ are the same number $R_{1}$.

(ii) Consider $\omega(r)=1-3 r, 0 \leq r<1$. The number $R_{2}=$ $(1-\alpha) / 3<1 / 3$ is clearly the root of the equation $\omega(r)=\alpha$. Since $\omega$ is decreasing, then $\omega(r) \geq \omega\left(R_{2}\right)=$ $\alpha$ for $0<r \leq R_{2}$. It follows from (23) that for $0<r=|z| \leq R_{2}$,

$$
\begin{gathered}
\operatorname{Re} \frac{z f^{\prime}(z)}{f(z)} \geq 1-3 r=\omega(r) \geq \alpha, \\
\left|\frac{z f^{\prime}(z)}{f(z)}-1\right| \leq 3 r=1-\omega(r) \leq 1-\alpha .
\end{gathered}
$$

Evaluating the function $f_{2}(z)=z e^{3 z}$ at $z=-R_{2}$ yields

$$
\frac{z f_{2}^{\prime}(z)}{f_{2}(z)}=1-3 R_{2}=\alpha \text {. }
$$

Hence,

$$
\begin{gathered}
\operatorname{Re} \frac{z f_{2}^{\prime}(z)}{f_{2}(z)}=\alpha, \\
\left|\frac{z f_{2}^{\prime}(z)}{f_{2}(z)}-1\right|=1-\alpha .
\end{gathered}
$$

This proves that the $\mathcal{S}^{*}(\alpha)$ and $\mathcal{S}_{\alpha}^{*}$ radii for the class $\mathscr{T}_{2}$ are the same number $R_{2}$.

Next, we find the $\mathcal{S}^{*}[A, B]$-radius (Janowski starlikeness) for $\mathscr{T}_{1}$ and $\mathscr{T}_{2}$. Recall that $\mathcal{S}^{*}[A, B]$ consists of analytic functions $f \in \mathscr{A}$ satisfying the subordination $z f^{\prime}(z) / f(z) \prec(1+A z) /(1+B z),-1 \leq B<A \leq 1$.

\section{Theorem 2.}

(i) Every $f \in \mathscr{T}_{1}$ is Janowski starlike in the disc $\mathbb{D}_{r}=$ $\{z:|z|<r\}$ for $r \leq 2(A-B) /(3(1+|B|)+2(A-B))$. If $B<0$, then $R_{\mathcal{S}^{*}[A, B]}\left(\mathscr{T}_{1}\right)=2(A-B) /$ $(3+2 A-5 B))$

(ii) The radius of Janowski starlikeness for $\mathscr{T}_{2}$ is $R_{\mathcal{S}^{*}[A, B]}\left(\mathscr{T}_{2}\right)=(A-B) /(3(1+|B|))$.

Proof. Since $\mathcal{S}^{*}[A,-1]=\mathcal{S}^{*}((1-A) / 2)$, the results in the case $B=-1$ follow from Theorem 1 . We now prove the results when $-1<B<A \leq 1$.

(i) Let $f \in \mathscr{T}_{1}$ and write $w=z f^{\prime}(z) / f(z)$. Then, (12) shows that $|w-1| \leq 3 r /(2(1-r))$ for $|z| \leq r$. For $0 \leq r \leq R_{1}:=2(A-B) /(3(1+|B|)+2(A-B))$, then $3 R_{1} /\left(2\left(1-R_{1}\right)\right)=(A-B) /(1+|B|)$.

For $0 \leq r \leq R_{1}$, we first show that the disc

$$
\left\{w:|w-1| \leq \frac{3 R_{1}}{2\left(1-R_{1}\right)}=\frac{A-B}{1+|B|}\right\}
$$

is contained in the images of the unit disc under the mapping $(1+A z) /(1+B z)$. As $B \neq-1$, the image is the disc given by 


$$
\left\{w:\left|w-\frac{1-A B}{1-B^{2}}\right|<\frac{A-B}{1-B^{2}}\right\}
$$

Silverman ([12], p. 50-51) has shown that the disc

$$
\{w:|w-c|<d\} \subset\{w:|w-a|<b\},
$$

if and only if $|a-c| \leq b-d$. With the choices $c=1$, $d=(A-B) /(1+|B|), \quad a=(1-A B) /\left(1-B^{2}\right)$, and $b=(A-B) /\left(1-B^{2}\right)$, then $|a-c|=|B|(A-B) /$ $\left(1-B^{2}\right)=b-d$. This proves that $\mathcal{S}^{*}[A, B]$ radius is at least $R_{1}$.

To prove sharpness, consider the function $f_{1} \in \mathscr{T}_{1}$ given by $f_{1}(z)=z(1+z)^{3 / 2}$. Evidently, $z f_{1}^{\prime}(z) / f_{1}$ $(z)=(2+5 z) /(2+2 z)$. For $\quad B<0$, evaluating at $z=-R_{1}$, then $z f_{1}^{\prime}(z) / f_{1}(z)=1+3 z /(2+2 z)=$ $1-(A-B) /(1+|B|)=(1-A) /(1-B)$. This shows that

$$
\left|\frac{z f_{1}^{\prime}(z)}{f_{1}(z)}-\frac{1-A B}{1-B^{2}}\right|=\left|\frac{1-A}{1-B}-\frac{1-A B}{1-B^{2}}\right|=\frac{A-B}{1-B^{2}},
$$

proving sharpness in the case $B<0$.

(ii) Let $f \in \mathscr{T}_{2}$ and $w:=z f^{\prime}(z) / f(z)$. It follows from (23) that $|w-1| \leq 3 r$ for $|z| \leq r$. For $0 \leq r \leq R_{2}:=(A-B) /(3(1+|B|))$, we see that the disc $\left\{w:|w-1| \leq 3 R_{2}=(A-B) /(1+|B|)\right\}$ is contained in the disc $\{w: \mid w-(1-A B) /$ $\left.\left(1-B^{2}\right) \mid<(A-B) /\left(1-B^{2}\right)\right\}$, as in the proof of (i). This proves that $\mathcal{S}^{*}[A, B]$ radius is at least $R_{2}$. The result is sharp for the function $f_{2} \in \mathscr{T}_{2}$ given by the function $f_{2}(z)=z e^{3 z}$.

The function $\varphi_{\mathrm{PAR}}: \mathbb{D} \longrightarrow \mathbb{C}$ given by

$$
\varphi_{\mathrm{PAR}}(z):=1+\frac{2}{\pi^{2}}\left(\log \frac{1+\sqrt{z}}{1-\sqrt{z}}\right)^{2}, \quad \operatorname{IM} \sqrt{z} \geq 0,
$$

maps $\mathbb{D}$ into the parabolic region

$$
\varphi_{\mathrm{PAR}}(\mathbb{D})=\left\{w=u+i v: v^{2}<2 u-1\right\}=\{w: \mathrm{RE} w>|w-1|\} .
$$

The class $\quad \mathscr{C}\left(\varphi_{\mathrm{PAR}}\right)=\left\{f \in \mathscr{A}: 1+z f^{\prime \prime}(z) / f^{\prime}(z)\right.$ $\left\langle\varphi_{\mathrm{PAR}}(z)\right\}$ is the class of uniformly convex functions introduced by Goodman [13]. The corresponding class $\mathcal{S}_{p}^{*}:=\mathcal{S}^{*}\left(\varphi_{\mathrm{PAR}}\right)=\left\{f \in \mathscr{A}: z f^{\prime}(z) / f(z)<\varphi_{\mathrm{PAR}}(z)\right\}$ introduced by Rønning [14] is known as the class of parabolic starlike functions. The class $\mathcal{S}_{p}^{*}$ consists of functions $f \in \mathscr{A}$ satisfying

$$
\operatorname{Re}\left(\frac{z f^{\prime}(z)}{f(z)}\right)>\left|\frac{z f^{\prime}(z)}{f(z)}-1\right|, \quad z \in \mathbb{D} .
$$

Evidently, every parabolic starlike function is also starlike of order $1 / 2$. The radius of parabolic starlikeness for the classes $\mathscr{T}_{1}$ and $\mathscr{T}_{2}$ is given in the next result.
Corollary 1. The radius of parabolic starlikeness for $\mathscr{T}_{1}$ and $\mathscr{T}_{2}$ is respectively equal to its radius of starlikeness of order $1 /$ 2. Thus,

$$
\begin{aligned}
\text { (i) } R_{\mathcal{S}_{p}^{*}}\left(\mathscr{T}_{1}\right) & =1 / 4 \\
\text { (ii) } R_{\mathcal{S}_{p}^{*}}\left(\mathscr{T}_{2}\right) & =1 / 6
\end{aligned}
$$

Proof. Shanmugam and Ravichandran ([15], p. 321) proved that

$$
\left\{w:|w-a|<a-\frac{1}{2}\right\} \subseteq\{w: \operatorname{RE} w>|w-1|\},
$$

for $1 / 2<a \leq 3 / 2$. Choosing $a=1$, this implies that $\mathcal{S}_{1 / 2}^{*} \subset \mathcal{S}_{p}^{*}$. Every parabolic starlike function is also starlike of order $1 / 2$, whence the inclusion $\delta_{1 / 2}^{*} \subset \mathcal{S}_{p}^{*} \subset \mathcal{S}^{*}(1 / 2)$. Therefore, for any class $\mathscr{F}$, readily $R_{\mathcal{S}_{1 / 2}^{*}}(\mathscr{F}) \leq R_{\mathcal{S}_{p}^{*}}$ $(\mathscr{F}) \leq R_{\mathcal{S}^{*}(1 / 2)}(\mathscr{F})$.

When $\mathscr{F}=\mathscr{T}_{i}, i=1,2$, Theorem 1 gives $R_{\mathcal{S}^{*}(\alpha)}\left(\mathscr{T}_{i}\right)=$ $R_{\mathcal{S}_{\alpha}^{*}}\left(\mathscr{T}_{i}\right)$. This shows that $R_{\mathcal{S}_{1 / 2}^{*}}\left(\mathscr{T}_{i}\right)=R_{\mathcal{S}_{p}^{*}}\left(\mathscr{T}_{i}\right)=R_{\mathcal{S}^{*}}$ $(1 / 2)\left(\mathscr{T}_{i}\right)$. Since $R_{\mathcal{S}^{*}(1 / 2)}\left(\mathscr{T}_{1}\right)=1 / 4$ and $R_{\mathcal{S}^{*}(1 / 2)}\left(\mathscr{T}_{2}\right)=1 / 6$ from Theorem 1, it follows that $R_{\delta_{p}^{*}}\left(\mathscr{T}_{1}\right)=1 / 4$ and $R_{\mathcal{S}_{p}^{*}}\left(\mathscr{T}_{2}\right)=1 / 6$.

\section{Further Radius of Starlikeness}

In this section, we find the $\mathscr{G}$-radius for the class $\mathscr{T}_{i}$ with $i=1,2$, for certain other widely studied subclasses $\mathscr{G}$. These are associated with particular choices of the superordinate function $\varphi$ in (27).

Denote by $\mathcal{S}_{\exp }^{*}:=\mathcal{S}^{*}\left(e^{z}\right)$ the class associated with $\varphi(z):=e^{z}$ in (27). This class was introduced by Mendiratta et al. [16], and it consists of functions $f \in \mathscr{A}$ satisfying the condition $\left|\log \left(z f^{\prime}(z) / f(z)\right)\right|<1$. The following result gives the radius of exponential starlikeness for the classes $\mathscr{T}_{1}$ and $\mathscr{T}_{2}$.

Corollary 2. The $\mathcal{S}_{\exp }^{*}$-radius for the class $\mathscr{T}_{1}$ is

$$
R_{\mathcal{S}_{\text {exp }}^{*}}\left(\mathscr{T}_{1}\right)=\frac{(2-2 e)}{(2-5 e)} \approx 0.296475
$$

while that of $\mathscr{T}_{2}$ is

$$
R_{\mathcal{S}_{\exp }^{*}}\left(\mathscr{T}_{2}\right)=\frac{(e-1)}{3 e} .
$$

Proof. Mendiratta et al. ([16], Lemma 2.2) proved that

$$
\left\{w:|w-a|<a-\frac{1}{e}\right\} \subseteq\{w:|\log w|<1\},
$$

for $e^{-1} \leq a \leq\left(e+e^{-1}\right) / 2$, and this inclusion with $a=1$ gives $\mathcal{S}_{1 / e}^{*} \subset \mathcal{S}_{\text {exp }}^{*}$. It was also shown in ([16], Theorem 2.1 (i)) that $\mathcal{S}_{\exp }^{*} \subset \mathcal{S}^{*}(1 / e)$. Therefore, $\mathcal{S}_{1 / e}^{*} \subset \mathcal{S}_{\exp }^{*} \subset \mathcal{S}^{*}(1 / e)$, which, as a consequence of Theorem 1, established the result.

Corollary 3 investigates the radius of cardioid starlikeness for each class $\mathscr{T}_{1}$ and $\mathscr{T}_{2}$. The class $S_{C}^{*}:=\mathcal{S}^{*}\left(\varphi_{\mathrm{CAR}}\right)$, 
where $\varphi_{\mathrm{CAR}}(z)=1+4 z / 3+2 z^{2} / 3$ in (27), was introduced and studied in [17]. Descriptively, $f \in S_{C}^{*}$ provided $z f^{\prime}(z) / f(z)$ lies in the region bounded by the cardioid $\Omega_{C}:=\left\{w=u+i v:\left(9 u^{2}+9 v^{2}-18 u+5\right)^{2}-16\left(9 u^{2}+9 v^{2}-\right.\right.$ $6 u+1)=0\}$.

Corollary 3. The following are the $S_{C}^{*}$-radius for the classes $\mathscr{T}_{1}$ and $\mathscr{T}_{2}$ :

(i) $R_{S_{C}^{*}}\left(\mathscr{T}_{1}\right)=4 / 13$,

(ii) $R_{S_{C}^{*}}\left(\mathscr{T}_{2}\right)=2 / 9$.

Proof. Sharma et al. [17] proved that $\{w:|w-a|<a-1 / 3\} \subseteq \Omega_{C}$ for $1 / 3<a \leq 5 / 3$, and this inclusion with $a=1$ gives $\delta_{1 / 3}^{*} \subset \mathcal{S}_{C}^{*}$. Thus, $R_{\mathcal{S}_{1 / 3}^{*}}\left(\mathscr{T}_{i}\right) \leq R_{\mathcal{S}_{C}^{*}}\left(\mathscr{T}_{i}\right)$ for $i=1,2$. To complete the proof, we demonstrate $R_{\mathcal{S}_{C}^{*}}\left(\mathscr{T}_{i}\right) \leq R_{\mathcal{S}_{1 / 3}^{*}}\left(\mathscr{T}_{i}\right)$ for $i=1,2$.

(i) Evaluating the function $f_{1}(z)=z(1+z)^{3 / 2}$ at $z=$ $-R=-R_{S_{1 / 3}^{*}}\left(\mathscr{T}_{1}\right)=-4 / 13$ gives

$\frac{z f_{1}^{\prime}(z)}{f_{1}(z)}=\frac{2+5 z}{2+2 z}=\frac{2-5 R}{2-2 R}=\frac{1}{3}=\varphi_{\mathrm{CAR}}(-1)$.

Thus, $R_{S_{C}^{*}}\left(\mathscr{T}_{1}\right) \leq 4 / 13$.

(ii) Similarly, at $z=-R=-R_{S_{1 / 3}^{*}}\left(\mathscr{T}_{2}\right)=-2 / 9$, the function $f_{2}(z)=z e^{3 z}$ yields

$$
\frac{z f_{2}^{\prime}(z)}{f_{2}(z)}=1+3 z=1-3 R=\frac{1}{3}=\varphi_{\mathrm{CAR}}(-1) .
$$

This proves that $R_{S_{C}^{*}}\left(\mathscr{T}_{2}\right) \leq 2 / 9$.

In 2019, Cho et al. [18] studied the class $\mathcal{S}_{\sin }^{*}:=\mathcal{S}^{*}(1+$ $\sin z$ ) consisting of functions $f \in \mathscr{A}$ satisfying the condition $z f^{\prime}(z) / f(z)<1+\sin z$. We find the $\delta_{\sin }^{*}$-radius for the classes $\mathscr{T}_{1}$ and $\mathscr{T}_{2}$.

Corollary 4. The following are the $\mathcal{S}_{\sin ^{*}}$-radius for each class $\mathscr{T}_{1}$ and $\mathscr{T}_{2}$ :

(i) $R_{\mathcal{S}_{\sin ^{*}}}\left(\mathscr{T}_{1}\right)=2(\sin 1) /(3+2 \sin 1) \approx 0.35938$,

(ii) $R_{\mathcal{S}_{\text {sin }^{*}}}\left(\mathscr{T}_{2}\right)=(\sin 1) / 3$.

Proof. It was proved in [18] that $\{w:|w-a|<\sin 1-|a-1|\} \subseteq q(\mathbb{D})$ for $|a-1| \leq \sin 1$, where $q(z):=1+\sin z$. For $a=1$, this implies that $\mathcal{S}_{1-\sin 1}^{*} \subset \mathcal{S}_{\text {sin }^{*}}$. Thus, $R_{\mathcal{S}_{1-\sin 1}^{*}}\left(\mathscr{T}_{i}\right) \leq R_{\mathcal{S}_{\text {sin }}^{*}}\left(\mathscr{T}_{i}\right)$ for $i=1,2$. The proof is completed by demonstrating $R_{\mathcal{S}_{\text {sin }}^{*}}\left(\mathscr{T}_{i}\right) \leq R_{\mathcal{S}_{1-\text { sin } 1}^{*}}\left(\mathscr{T}_{i}\right)$ for $i=1,2$.

(i) Evaluating the function $f_{1}(z)=z(1+z)^{3 / 2}$ at $z=$ $-R=-R_{S_{1-\sin 1}^{*}}\left(\mathscr{T}_{1}\right)=-2 \sin 1 /(3+2 \sin 1)$ gives

$\frac{z f_{1}^{\prime}(z)}{f_{1}(z)}=\frac{2+5 z}{2+2 z}=\frac{2-5 R}{2-2 R}=1-\sin 1=q(-1)$.

Thus, $R_{\delta_{\sin ^{*}}}\left(\mathscr{T}_{1}\right) \leq 22 \sin 1 /(3+2 \sin 1)$. (ii) Similarly, at $z= \pm R= \pm R_{S_{1-\text { sin }}^{*}}\left(\mathscr{T}_{2}\right)=$ $\pm(\sin 1) / 3$, the function $f_{2}(z)=z e^{3 z}$ yields

$\frac{z f_{2}^{\prime}(z)}{f_{2}(z)}=1+3 z=1 \pm 3 R=1 \pm \sin 1=q( \pm 1)$.

This proves that $R_{\mathcal{S}_{\sin ^{*}}}\left(\mathscr{T}_{2}\right) \leq(\sin 1) / 3$.

Consider next the class $\mathcal{S}_{\odot}^{*}:=\mathcal{S}^{*}\left(z+\sqrt{1+z^{2}}\right)$ introduced by Raina and Sokół in [19]. Functions $f \in \mathcal{S}_{\odot}^{*}$ provided $z f^{\prime}(z) / f(z)$ lies in the region bounded by the lune $\Omega_{l}:=\left\{w:\left|w^{2}-1\right|<2|w|\right\}$. The result below gives the radius of lune starlikeness for each class $\mathscr{T}_{1}$ and $\mathscr{T}_{2}$.

Corollary 5. The following are the $\mathcal{S}_{\odot}^{*}$-radius for each class $\mathscr{T}_{1}$ and $\mathscr{T}_{2}$ :
(i) $R_{\mathcal{S}_{\oplus}^{*}}\left(\mathscr{T}_{1}\right)=2(\sqrt{2}-2) /(2 \sqrt{2}-7) \approx 0.280847$,
(ii) $R_{\mathcal{S}_{\oplus}^{*}}\left(\mathscr{T}_{2}\right)=(2-\sqrt{2}) / 3$.

Proof. It was shown by Gandhi and Ravichandran ([20], Lemma 2.1) that $\{w:|w-a|<1-|\sqrt{2}-a|\} \subseteq \Omega_{l}$ for $\sqrt{2}-1<a \leq \sqrt{2}+1$. Choosing $a=1$, the inclusion gives $\mathcal{S}_{\sqrt{2}-1}^{*} \subset \mathcal{S}_{\mathscr{\Theta}}^{*}$. Thus, $R_{\mathcal{S}^{*} \sqrt{2}-1}\left(\mathscr{T}_{i}\right) \leq R_{\mathcal{S}_{\odot}^{*}}\left(\mathscr{T}_{i}\right)$ for $i=1,2$. We complete the proof ${ }^{\sqrt{2}}$ by demonstrating $R_{\mathcal{S}_{\oplus}^{*}}\left(\mathscr{T}_{i}\right) \leq$ $R_{\mathcal{S}_{\sqrt{2}-1}^{*}}\left(\mathscr{T}_{i}\right)$ for $i=1,2$.

(i) Evaluating the function $f_{1}(z)=z(1+z)^{3 / 2}$ at $z=$ $-R=-R_{S_{\sqrt{2}-1}^{*}}\left(\mathscr{T}_{1}\right)=-2(\sqrt{2}-2) /(2 \sqrt{2}-7)$ gives

$$
\begin{aligned}
\left|\left(\frac{z f_{1}^{\prime}(z)}{f_{1}(z)}\right)^{2}-1\right| & =\left|\left(\frac{2+5 z}{2+2 z}\right)^{2}-1\right|=\left|\left(\frac{2-5 R}{2-2 R}\right)^{2}-1\right| \\
& =2(\sqrt{2}-1)=2\left|\frac{z f_{1}^{\prime}(z)}{f_{1}(z)}\right| .
\end{aligned}
$$

Thus, $R_{S_{\oplus}^{*}}\left(\mathscr{T}_{1}\right) \leq 2(\sqrt{2}-2) /(2 \sqrt{2}-7)$.

(ii) Similarly, at $z=-R=-R_{S^{*}}\left(\mathscr{T}_{2}\right)=-(2-\sqrt{2}) / 3$, the function $f_{2}(z)=z e^{3 z}$ yields

$$
\begin{aligned}
\left|\left(\frac{z f_{2}^{\prime}(z)}{f_{2}(z)}\right)^{2}-1\right| & =\left|(1+3 z)^{2}-1\right|=\left|(1-3 R)^{2}-1\right| \\
& =2(\sqrt{2}-1)=2\left|\frac{z f_{2}^{\prime}(z)}{f_{2}(z)}\right| .
\end{aligned}
$$

This proves that $R_{S_{\oplus}^{*}}\left(\mathscr{T}_{2}\right) \leq(2-\sqrt{2}) / 3$.

As a further example, consider next the class $\mathcal{S}_{R}^{*}:=\mathcal{S}^{*}(\eta(z))$, where $\eta(z)=1+\left(\left(z k+z^{2}\right) /\left(k^{2}-k z\right)\right)$, $k=\sqrt{2}+1$. This class associated with a rational function was introduced and studied by Kumar and Ravichandran in [21].

Corollary 6. The following are the $\mathcal{S}_{R}^{*}$-radius for the classes $\mathscr{T}_{1}$ and $\mathscr{T}_{2}$ : 
(i) $R_{\mathcal{S}_{R}^{*}}\left(\mathscr{T}_{1}\right)=2(-3+2 \sqrt{2}) /(4 \sqrt{2}-9) \approx 0.102642$,

(ii) $R_{\mathcal{S}_{R}^{*}}\left(\mathscr{T}_{2}\right)=(3-2 \sqrt{2}) / 3$.

Proof. It was shown in [21] that $\{w:|w-a|<a-2(\sqrt{2}-1)\} \subseteq \eta(\mathbb{D}) \quad$ for $\quad 2(\sqrt{2}-1)$ $<a \leq \sqrt{2}$. This inclusion with $a=1$ gives $\mathcal{S}_{2(\sqrt{2}-1)}^{*} \subset \mathcal{S}_{R}^{*}$. Thus, $R_{\mathcal{S}_{2(\sqrt{2}-1)}^{*}}\left(\mathscr{T}_{i}\right) \leq R_{\mathcal{S}_{R}^{*}}\left(\mathscr{T}_{i}\right)$ for $i=1,2$. We next show that $R_{\mathcal{S}_{R}^{*}}\left(\mathscr{T}_{i}\right) \leq R_{\mathcal{S}_{2(\sqrt{2}-1)}^{*}}\left(\mathscr{T}_{i}\right)$ for $i=1,2$.

(i) At $z=-R=-R_{S_{2(\sqrt{2}-1)}^{*}}\left(\mathscr{T}_{1}\right)=-2(-3+2 \sqrt{2})$ / $(4 \sqrt{2}-9)$, the function $f_{1}(z)=z(1+z)^{3 / 2}$ yields

$$
\frac{z f_{1}^{\prime}(z)}{f_{1}(z)}=\frac{2-5 R}{2-2 R}=2(\sqrt{2}-1)=\eta(-1) \text {. }
$$

Thus, $R_{\mathcal{S}_{R}^{*}}\left(\mathscr{T}_{1}\right) \leq 2(-3+2 \sqrt{2}) /(4 \sqrt{2}-9)$. (ii) Evaluating $f_{2}(z)=z e^{3 z}$ at $z=-R=-R_{S_{2(\sqrt{2}-1)}^{*}}$
$\left(\mathscr{T}_{2}\right)=-(3-2 \sqrt{2}) / 3$ gives

$$
\frac{z f_{2}^{\prime}(z)}{f_{2}(z)}=1-3 R=2(\sqrt{2}-1)=\eta(-1) .
$$

Thus, $R_{\mathcal{S}_{R}^{*}}\left(\mathscr{T}_{2}\right) \leq(3-2 \sqrt{2}) / 3$.

The class $\mathcal{S}_{N_{e}}^{*}:=\mathcal{S}^{*}(\psi(z))$, where $\psi(z)=1+z-z^{3} / 3$, was introduced and studied by Wani and Swaminathan in [22]. Geometrically, $f \in \mathcal{S}_{N_{e}}^{*}$ provided $z f^{\prime}(z) / f(z)$ lies in the region bounded by the nephroid: a 2-cusped kidneyshaped curve $\Omega_{N_{e}}:=\left\{w=u+i v:\left((u-1)^{2}+v^{2}-4 / 9\right)^{3}-\right.$ $\left.4 v^{2} / 3=0\right\}$.

Corollary 7. The following are the $\mathcal{S}_{N_{e}}^{*}$-radius for the classes $\mathscr{T}_{1}$ and $\mathscr{T}_{2}$ :

(i) $R_{\mathcal{S}_{N_{e}}^{*}}\left(\mathscr{T}_{1}\right)=4 / 13$,

(ii) $R_{\mathcal{S}_{N_{e}}^{*}}\left(\mathscr{T}_{2}\right)=2 / 9$.

Proof. It was shown in [22] that $\{w:|w-a|<a-1 / 3\} \subseteq \Omega_{N}$ for $1 / 3<a \leq 1$. This inclusion with $a=1$ gives $\mathcal{S}_{1 / 3}^{*} \subset \mathcal{S}_{N_{e}}^{*}$. This shows that $R_{\mathcal{S}_{1 / 3}^{*}}\left(\mathscr{T}_{i}\right)$ $\leq R_{\mathcal{S}_{N_{e}}^{*}}\left(\mathscr{T}_{i}\right)$ for $i=1,2$. We next show that $R_{\mathcal{S}_{N_{e}}^{*}}\left(\mathscr{T}_{i}\right)$ $\leq R_{\mathcal{S}_{1 / 3}^{*}}^{*}\left(\mathscr{T}_{i}\right)$ for $i=1,2$.

(i) Evaluating the function $f_{1}(z)=z(1+z)^{3 / 2}$ at $z=$ $-R=-R_{S_{1 / 3}^{*}}\left(\mathscr{T}_{1}\right)=-4 / 13$ results in

$$
\frac{z f_{1}^{\prime}(z)}{f_{1}(z)}=\frac{2-5 R}{2-2 R}=\frac{1}{3}=\psi(-1) \text {. }
$$

Thus, $R_{S_{N_{e}}^{*}}\left(\mathscr{T}_{1}\right) \leq 4 / 13$.

(ii) Similarly, evaluating $f_{2}(z)=z e^{3 z}$ at $z=-R=$ $-R_{S_{1 / 3}^{*}}\left(\mathscr{T}_{2}\right)=-2 / 9$ yields

$$
\frac{z f_{2}^{\prime}(z)}{f_{2}(z)}=1-3 R=\frac{1}{3}=\psi(-1) .
$$

This proves that $R_{S_{N_{e}}^{*}}\left(\mathscr{T}_{2}\right) \leq 2 / 9$.

Finally, we consider the class $\mathcal{S}_{\mathrm{SG}}^{*}:=\mathcal{S}^{*}\left(2 /\left(1+e^{-z}\right)\right)$ introduced by Goel and Kumar in [23]. Here, $2 /\left(1+e^{-z}\right)$ is the modified sigmoid function that maps $\mathbb{D}$ onto the region $\left.\Omega_{\mathrm{SG}}:=w=u+i v:|\log (w /(2-w))|<1\right\}$. Thus, $f \in \mathcal{S}_{\mathrm{SG}}^{*}$ provided the function $z f^{\prime}(z) / f(z)$ maps $\mathbb{D}$ onto the region lying inside the domain $\Omega_{\mathrm{SG}}$.

Corollary 8. The $\mathcal{S}_{S G}^{*}$-radius for the class $\mathscr{T}_{1}$ is

$$
R_{\mathcal{S}_{\mathrm{SG}}^{*}}\left(\mathscr{T}_{1}\right)=\frac{(2 e-2)}{(1+5 e)} \approx 0.23552,
$$

while that of $\mathscr{T}_{2}$ is

$$
R_{\mathcal{S}_{\mathrm{SG}}^{*}}\left(\mathscr{T}_{2}\right)=\frac{(e-1)}{(3(1+e))} .
$$

Proof. The inclusion $\{w:|w-a|<((e-1) /(e+1))-\mid a-$ $1 \mid\} \subseteq \Omega_{\mathrm{SG}}$ holds for $2 /(1+e)<a<2 e /(1+e)$ (see [23]). At $a=1$, the set inclusion shows that $\mathcal{S}_{2 /(e+1)}^{*} \subset \mathcal{S}_{\mathrm{SG}}^{*}$. It was also shown in [23] that $\mathcal{S}_{\mathrm{SG}}^{*} \subset \mathcal{S}^{*}(\alpha)$ for $0 \leq \alpha \leq 2 /(e+1)$. The desired result is now an immediate consequence of Theorem 1 .

\section{Data Availability}

No data were used to support this study.

\section{Conflicts of Interest}

The authors declare that they have no conflicts of interest.

\section{Acknowledgments}

The first author gratefully acknowledges the support from USM research university grant 1001.PMATHS.8011101. A preprint of an earlier version is available at https://arxiv.org/ abs/2101.01617.

\section{References}

[1] P. L. Duren, Univalent Functions, GTM 259, Springer-Verlag, New York, NY, USA, 1983.

[2] W. Kaplan, "Close-to-convex schlicht functions," Michigan Mathematical Journal, vol. 1, pp. 169-185, 1952.

[3] M. O. Reade, "On close-to-close univalent functions," Michigan Mathematical Journal, vol. 3, pp. 59-62, 1955.

[4] T. H. MacGregor, "The radius of convexity for starlike functions of order (1/2)," Proceedings of the American Mathematical Society, vol. 14, pp. 71-76, 1963.

[5] T. H. MacGregor, "The radius of univalence of certain analytic functions," Proceedings of the American Mathematical Society, vol. 14, pp. 514-520, 1963.

[6] T. H. MacGregor, "The radius of univalence of certain analytic functions II," Proceedings of the American Mathematical Society, vol. 14, pp. 521-524, 1963.

[7] T. H. MacGregor, "A class of univalent functions," Proceedings of the American Mathematical Society, vol. 15, pp. 311-317, 1964. 
[8] J. S. Ratti, "The radius of univalence of certain analytic functions," Mathematische Zeitschrift, vol. 107, pp. 241-248, 1968.

[9] J. S. Ratti, "The radius of convexity of certain analytic functions," Indian Journal of Pure and Applied Mathematics, vol. 1, no. 1, pp. 30-36, 1970.

[10] A. Lecko, V. Ravichandran, and A. Sebastian, "Starlikeness of certain non-univalent functions," Analysis and Mathematical Physics, vol. 11, p. 163, 2021.

[11] A. Sebastian and V. Ravichandran, "Radius of starlikeness of certain analytic functions," Mathematica Slovaca, vol. 71, no. 1, pp. 83-104, 2021.

[12] H. Silverman and E. M. Silvia, "Subclasses of starlike functions subordinate to convex functions," Canadian Journal of Mathematics, vol. 37, no. 1, pp. 48-61, 1985.

[13] A. W. Goodman, Univalent Functions, Vol. 2, Mariner, Tampa, FL, USA, 1983.

[14] F. Rønning, "Uniformly convex functions and a corresponding class of starlike functions," Proceedings of the American Mathematical Society, vol. 118, no. 1, pp. 189-196, 1993.

[15] T. N. Shanmugam and V. Ravichandran, "Certain properties of uniformly convex functions," Computational Methods and Function Theory 1994 (Penang), 319-324, Ser. Approx. Decompos., 5, World Scientific Publishing, River Edge, NJ, USA, 1994.

[16] R. Mendiratta, S. Nagpal, and V. Ravichandran, "On a subclass of strongly starlike functions associated with exponential function," Bulletin of the Malaysian Mathematical Sciences Society, vol. 38, no. 1, pp. 365-386, 2015.

[17] K. Sharma, N. K. Jain, and V. Ravichandran, "Starlike functions associated with a cardioid," Afrika Matematika, vol. 27, no. 5, pp. 923-939, 2016.

[18] N. E. Cho, V. Kumar, S. S. Kumar, and V. Ravichandran, "Radius problems for starlike functions associated with the sine function," Bulletin of the Iranian Mathematical Society, vol. 45, no. 1, pp. 213-232, 2019.

[19] R. K. Raina and J. Sokół, "Some properties related to a certain class of starlike functions," Comptes rendus de l'Académie des Sciences, vol. 353, no. 11, pp. 973-978, 2015.

[20] S. Gandhi, Ravichandran, and V. Starlike, "Functions associated with a lune," Asian-European Journal of Mathematics, vol. 10, no. 4, p. 12, Article ID 1750064, 2017.

[21] S. Kumar and V. Ravichandran, "A subclass of starlike functions associated with a rational function," Southeast Asian Bulletin of Mathematics, vol. 40, no. 2, pp. 199-212, 2016.

[22] L. A. Wani and A. Swaminathan, "Starlike and convex functions associated with a nephroid domain," Bulletin of the Malaysian Mathematical Sciences Society, vol. 44, 2020.

[23] P. Goel and S. Sivaprasad Kumar, "Certain class of starlike functions associated with modified sigmoid function," Bulletin of the Malaysian Mathematical Sciences Society, vol. 43, no. 1, pp. 957-991, 2020. 